\title{
Sucrose- and $\mathrm{H}^{+}$-Dependent Charge Movements Associated with the Gating of Sucrose Transporter ZmSUT1
}

\author{
Armando Carpaneto ${ }^{1}$, Hermann Koepsell ${ }^{2}$, Ernst Bamberg ${ }^{3}$, Rainer Hedrich ${ }^{4}$, Dietmar Geiger ${ }^{4 *}$
}

1 Istituto di Biofisica-CNR, Genova, Italy, 2 Institut für Anatomie und Zellbiologie, Universität Würzburg, Würzburg, Germany, 3 Max-Plank-Institute for Biophysics, Frankfurt am Main, Germany, 4 Julius-von-Sachs-Institut, Molekulare Pflanzenphysiologie und Biophysik, Universität Würzburg, Würzburg, Germany

\begin{abstract}
Background: In contrast to man the majority of higher plants use sucrose as mobile carbohydrate. Accordingly protondriven sucrose transporters are crucial for cell-to-cell and long-distance distribution within the plant body. Generally very negative plant membrane potentials and the ability to accumulate sucrose quantities of more than $1 \mathrm{M}$ document that plants must have evolved transporters with unique structural and functional features.

Methodology/Principal Findings: To unravel the functional properties of one specific high capacity plasma membrane sucrose transporter in detail, we expressed the sucrose $/ \mathrm{H}^{+}$co-transporter from maize ZmSUT1 in Xenopus oocytes. Application of sucrose in an acidic $\mathrm{pH}$ environment elicited inward proton currents. Interestingly the sucrose-dependent $\mathrm{H}^{+}$ transport was associated with a decrease in membrane capacitance $\left(C_{m}\right)$. In addition to sucrose $C_{m}$ was modulated by the membrane potential and external protons. In order to explore the molecular mechanism underlying these $C_{m}$ changes, presteady-state currents $\left(I_{\text {pre }}\right)$ of ZmSUT1 transport were analyzed. Decay of $I_{\text {pre }}$ could be best fitted by double exponentials. When plotted against the voltage the charge $Q$, associated to $I_{\text {pre, }}$ was dependent on sucrose and protons. The mathematical derivative of the charge $Q$ versus voltage was well in line with the observed $C_{m}$ changes. Based on these parameters a turnover rate of 500 molecules sucrose/s was calculated. In contrast to gating currents of voltage dependentpotassium channels the analysis of ZmSUT1-derived presteady-state currents in the absence of sucrose $(I=Q / \tau)$ was sufficient to predict ZmSUT1 transport-associated currents.

Conclusions: Taken together our results indicate that in the absence of sucrose, 'trapped' protons move back and forth between an outer and an inner site within the transmembrane domains of ZmSUT1. This movement of protons in the electric field of the membrane gives rise to the presteady-state currents and in turn to $C_{m}$ changes. Upon application of external sucrose, protons can pass the membrane turning presteady-state into transport currents.
\end{abstract}

Citation: Carpaneto A, Koepsell H, Bamberg E, Hedrich R, Geiger D (2010) Sucrose- and $\mathrm{H}^{+}$-Dependent Charge Movements Associated with the Gating of Sucrose Transporter ZmSUT1. PLoS ONE 5(9): e12605. doi:10.1371/journal.pone.0012605

Editor: Karin Schumacher, University of Heidelberg, Germany

Received June 22, 2010; Accepted August 3, 2010; Published September 7, 2010

Copyright: (c) 2010 Carpaneto et al. This is an open-access article distributed under the terms of the Creative Commons Attribution License, which permits unrestricted use, distribution, and reproduction in any medium, provided the original author and source are credited.

Funding: This work was supported by the Deutsche Forschungsgemeinschaft (DFG, http://www.dfg.de/index.jsp) grants within SFB487 to RH and HK. DG was supported by DFG grant GE2195/1-1. AC was supported by Italian Consiglio Nazionale delle Ricerche (CNR, Short-term Mobility program 2007 and 2009, http:// www.cnr.it/sitocnr/Englishversion/CNR/Dataandstatistics/International-activities/Shorttermmobilityprogram.html). The funders had no role in study design, data collection and analysis, decision to publish, or preparation of the manuscript.

Competing Interests: The authors have declared that no competing interests exist.

* E-mail: geiger@botanik.uni-wuerzburg.de

\section{Introduction}

For long distance transport from the side of production (source) in leaves to the user (sink) tissues, sucrose is loaded into the tubelike phloem network [1]. Phloem loading of sucrose, synthesized in photosynthetic cells (mesophyll) within the leaves, takes place in the sieve tube adjacent to companion cells. These transport-active cells appear to be interconnected via plasmodesmata to the sieve tubes. The flux and direction of sucrose is regulated by SUC/SUT type sucrose cotransporters [2,3,4,5,6]. Plant and animal sugar carriers shuttle their substrates in cotransport with protons or sodium ions, respectively. In contrast to animal cells, plants cells establish a $\mathrm{pH}$ gradient (acidic extracellular space) and very negative membrane potentials via plasma membrane proton pumps. From this electromotive force sucrose transporters gain energy to drive sucrose accumulation of more than $1 \mathrm{M}$. Recently detailed biophysical studies of ZmSUT1 revealed that this carrier is working like a perfect thermodynamic machine by which the proton gradient drives sucrose transport and vice versa on the basis of a $1: 1 \mathrm{H}^{+}$:sucrose stoichiometry [7]. As a matter of fact ZmSUT1 is capable to mediate sucrose loading and unloading of the phloem [6] under physiological conditions. The ZmSUT1 behavior is in contrast to the animal counterpart SGLT1, which mediates sugar uptake only. These fundamental physiological differences between plant phloem- and animal blood stream sugar transporters are harbored in their unique structure-function relationships.

The knowledge about the transport cycle of plant sucrose transporters is, however, still very limited and dates back to the 1990s $[8,9]$. Cotransporters characteristically display three main 
kinds of electrical activity. Besides the membrane current associated with the ion-coupled translocation of the organic substrate (transport-associated current, $\mathrm{I}_{\mathrm{tr}}$ ), most cotransporters exhibit two further kinds of current in the absence of organic substrate: an 'uncoupled' (steady) current and a presteady-state current $\left(\mathrm{I}_{\text {pre }}\right)[10,11,12,13]$. While the presteady-state current is best observed in the absence of substrate, it disappears when the substrate is present in saturating amounts [14,15]. Using presteady-state measurements and voltage clamp fluorometry the Wright lab $[11,16,17,18]$ examined the transport cycle of the $\mathrm{Na}^{+}$ driven glucose cotransporter SGLT1 during sugar transport. They recorded changes in charge movement in response to rapid membrane potential jumps in the presence and absence of sugar. In $\mathrm{Na}^{+}$buffers and in the absence of glucose, stepwise jumps in the membrane voltage elicited presteady-state currents (charge movements). Application of glucose, however, induced transportassociated inward $\mathrm{Na}^{+}$currents and reduced the maximal charge movements $\left(Q_{\max }\right)$. Presteady-state currents were completely inhibited by saturating sugar concentrations. Based on their results the authors developed an ordered eight-state model for the transport mechanism of SGLT1. Therein charge movements of SGLT1, giving rise to the observed presteady-state currents, were shown to be associated with the binding of sodium to the empty transporters (e.g. $[16,18])$.

In addition to the conductance, the capacitance of biological membranes $\left(\mathrm{C}_{\mathrm{m}}\right)$ represents a basic electrical property. Changes in $\mathrm{C}_{\mathrm{m}}$ arise from changes of the membrane surface area (endo- or exocytosis) or charge displacement in the electrical field of the membrane. The latter occurs e.g. when charged domains of integral membrane proteins undergo conformational changes or when ions bind to the protein within the electrical field of the membrane [19,20,21,22,23,24,25]. Thus charge displacements can turn membranes into highly nonlinear, time-varying capacitors. In this respect membrane proteins can be seen as insulators with specific dielectric properties and not only as conductors for ions/substrates.

In this study we combined $\mathrm{C}_{\mathrm{m}}$ and presteady-state current measurements to elucidate the transport mechanism of ZmSUT1 in Xenopus oocytes. $\mathrm{C}_{\mathrm{m}}$ measurements at various sucrose concentrations revealed a dose-dependent, reversible decrease of $\mathrm{C}_{\mathrm{m}}$. Furthermore $\mathrm{C}_{\mathrm{m}}$ was modulated by the membrane potential and the external proton concentration. To explore the molecular mechanism underlying the $\mathrm{C}_{\mathrm{m}}$ changes, presteady-state currents
( $\mathrm{I}_{\text {pre }}$ ) of ZmSUT1 transport were measured. Thereby we observed pronounced charge movements in the absence of the substrate. The decay rate constant $(\tau)$ of $\mathrm{I}_{\text {pre }}$ and the quantity of displaced charges $(Q)$ depended on the voltage and proton concentration. The quantitative comparison of transport-associated currents and $I_{\text {pre }}$ revealed that sucrose-induced transport currents can be predicted solely by the analysis of $\mathrm{I}_{\text {pre }}$ in the absence of sucrose. This relation suggests that charge movements at zero sucrose and sucrose-induced transport of protons arise from the same molecular mechanism.

\section{Results}

\section{Ligands Induce Voltage Dependent Capacitance Changes in ZmSUT1}

The expression of ZmSUT1 in Xenopus oocytes and the application of $0.5,1,3$ and $100 \mathrm{mM}$ sucrose to the external medium induced dose-dependent inward currents (see Fig. 1 [7]). In addition to the current recorded via the two-electrode voltageclamp technique (TECV) membrane capacitance changes were monitored [26]. In the absence of external sucrose at $\mathrm{V}=-20 \mathrm{mV}, \mathrm{C}_{\mathrm{m}}$ for the depicted, representative oocyte was $190 \mathrm{nF}$. Upon addition of sucrose the membrane capacitance decreased reversibly in a dose-dependent manner (Fig. 1A). Upon application of external sucrose the membrane capacitance decreased at all voltages tested. In water injected oocytes $\mathrm{C}_{\mathrm{m}}$ was not influenced by the addition of sucrose (data not shown). In absence of sucrose, voltage-dependent $\mathrm{C}_{\mathrm{m}}$ changes were characterized by a bell-shaped curve with a maximum around $-80 \mathrm{mV}$ (Fig. 1B). During a stepwise increase in sucrose concentrations, the $\mathrm{C}_{\mathrm{m}}$ drop saturated above $3 \mathrm{mM}$ of the disaccharide at $\mathrm{pH} 4$. ZmSUT1 represents a proton/sucrose cotransporter that drives sucrose at the expense of the proton gradient and vice versa [7]. We, thus, investigated whether and how external protons modulate $\mathrm{C}_{\mathrm{m}}$. With the representative oocyte clamped to $-20 \mathrm{mV}$ and sucrose-free bath solution buffered to $\mathrm{pH} 4, \mathrm{C}_{\mathrm{m}}$ was $178 \mathrm{nF}$ (Fig. 2A). A decrease in the proton concentration from $\mathrm{pH} 4$ to $\mathrm{pH} 5,6$, and 7 was followed by a drop in capacitance characterized by a maximum at $\mathrm{pH}$. The significant decrease of $\mathrm{C}_{\mathrm{m}}$ upon a shift from $\mathrm{pH} 4$ to $\mathrm{pH} 3$, equivalent to $1 \mathrm{mM}$ protons, was less pronounced than a similar shift of one $\mathrm{pH}$ unit from 4 to 5 . The voltage-dependence of the membrane capacitance at different $\mathrm{pH}$ values appeared nonlinear (Fig. 2B).

A
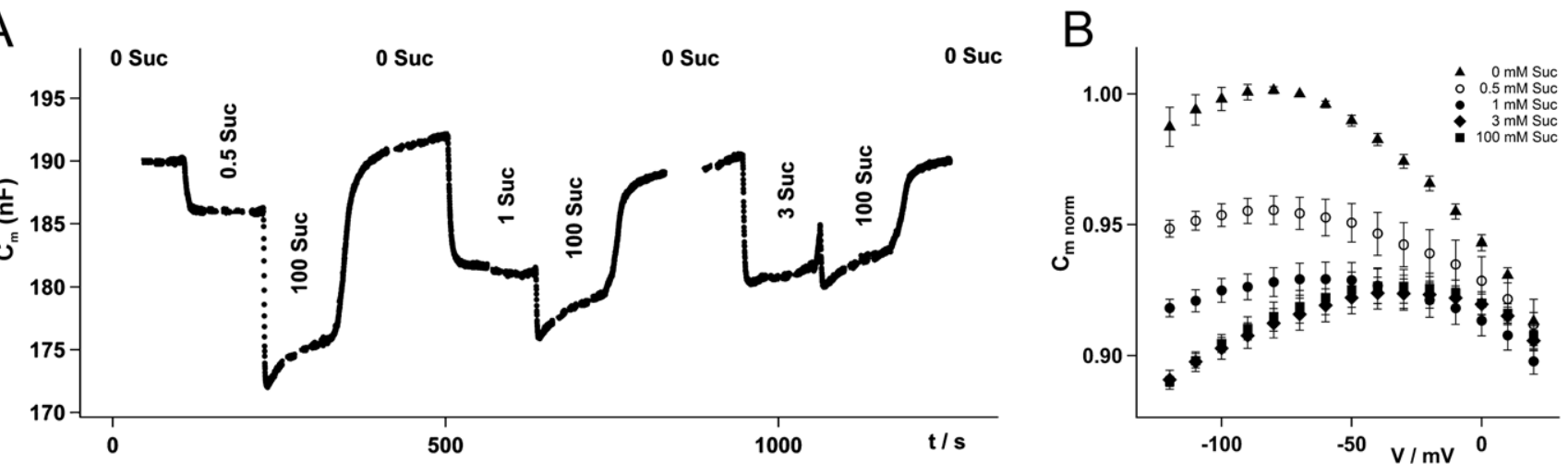

Figure 1. Sucrose dependent membrane capacitance changes in ZmSUT1 expressing oocytes. A) While continuously recording the membrane capacitance $\left(\mathrm{C}_{\mathrm{m}}\right)$ at $-20 \mathrm{mV}$ and $\mathrm{pH} 4$, various external solutions (indicated in the figure) were applied. Upon perfusion with sucrose containing external media the $C_{m}$ decreased in a dose dependent manner. B) When plotting $C_{m}$ against the membrane potential it became apparent that at all tested voltages, $\mathrm{C}_{\mathrm{m}}$ decreased in the presence of sucrose in a dose dependent manner (standard solution pH 4 , sucrose concentrations are indicated in the figure, $n \geq 4, \pm S D)$.

doi:10.1371/journal.pone.0012605.g001 
A

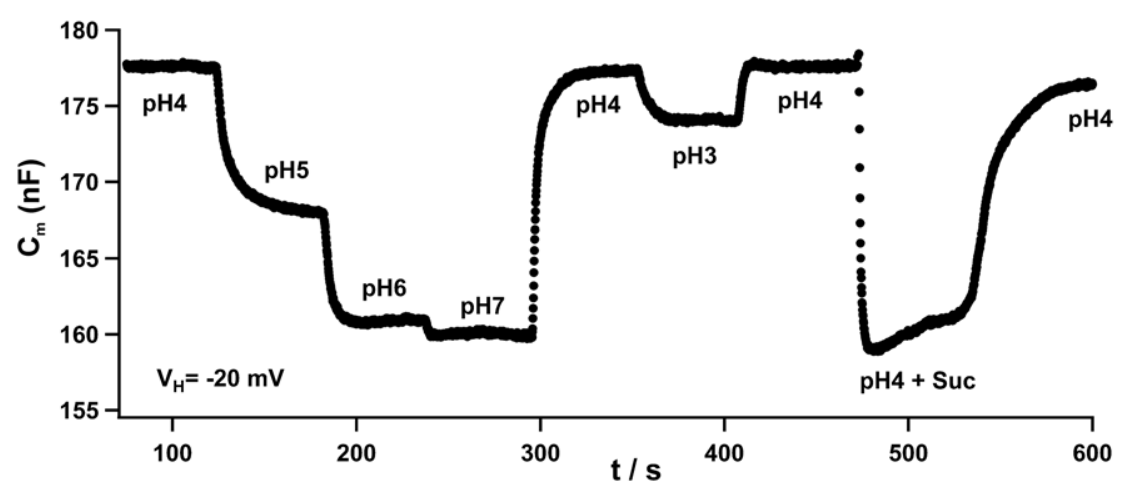

B

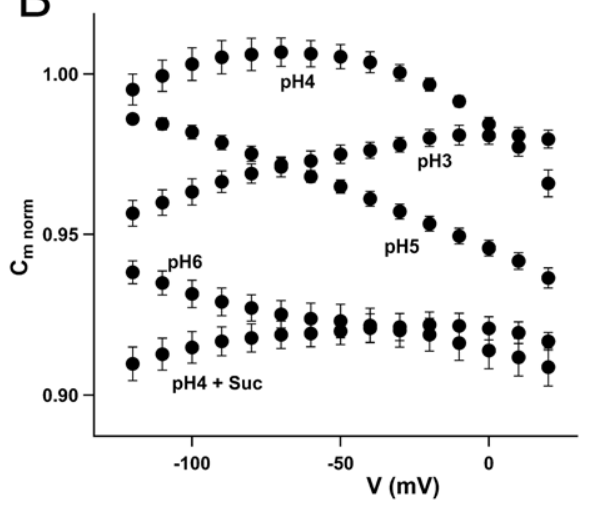

Figure 2. Capacitance changes in response to varying proton concentrations. A) Continuous recording of $C_{m}$ at $-20 \mathrm{mV}$ and indicated $\mathrm{pH}$ values. When exchanging the bath solution by stepwise decreasing proton concentrations from $\mathrm{pH} 4$ to 7 in the absence of sucrose, $\mathrm{C}_{\mathrm{m}}$ decreased reversibly. Increasing the proton concentration from pH 4 to 3 at $-20 \mathrm{mV}$ led to a decrease of $C_{m}$ as well. Subsequent application of saturating sucrose concentrations decreased $C_{m}$ to similar levels as in the presence of neutral buffers. B) $C_{m} /$ voltage plot at various pH values without sucrose and at $\mathrm{pH} 4$ in the presence of sucrose. Decreasing the proton concentration resulted in a shift of the $C_{m}$ peak towards more negative membrane potentials. At $\mathrm{pH} 4$ and in the presence of saturating sucrose concentrations $C_{m}$ was depressed at all voltages tested $(n \geq 5, \pm S D)$. $C_{m}$ values were normalized to values at $\mathrm{pH} 4$ in the absence of sucrose.

doi:10.1371/journal.pone.0012605.g002

For a wide voltage range, from -120 to $0 \mathrm{mV}, \mathrm{C}_{\mathrm{m}}$ peaked at $\mathrm{pH} 4$ dropping at both lower (pH 5 and 6) and higher (pH 3) external proton concentrations. Moreover at $\mathrm{pH} 5$ when compared to $\mathrm{pH} 3$ an opposite voltage-dependence was recorded. Upon depolarization $\mathrm{C}_{\mathrm{m}}$ decreased at $\mathrm{pH} 5$ while it increased at $\mathrm{pH} 3$. The application of saturating sucrose concentration lowered $\mathrm{C}_{\mathrm{m}}$ at all voltages. In order to interpret the results obtained so far, we assumed that changes in membrane capacitance in ZmSUT1 expressing oocytes were associated with charge movements inside the transporter.

\section{Charge Movements in ZmSUT1 Generate Presteady-State Currents}

Charge movements (presteady-state currents) inside membrane proteins have been observed with ion channels [27]. These 'presteady-state- or gating currents' precede the opening and closing of voltage-dependent channels. To investigate the molecular basis of sugar-dependent modulation of $\mathrm{C}_{\mathrm{m}}$ with ZmSUT1, we monitored voltage-dependent presteady-state currents of the sugar/proton co-transporter with high resolution. Thereby currents in response to membrane potential changes from a holding potential of $-20 \mathrm{mV}$ to $-80 \mathrm{mV}$ in the absence and presence of saturating sucrose concentration were compared (inset of Fig. 3A). As a matter of fact the stationary current in the presence of sucrose was significantly higher than in the absence of the sugar. These stationary sucrose-induced currents were named transport-associated currents $\left(\mathrm{I}_{\mathrm{tr}}\right)$. The transient current right after the voltage jump in the absence of sucrose, appeared slower in reaching the stationary level than with sucrose. This is better visible when subtracting the stationary currents (Fig. 3A). Presteady-state currents ( $\mathrm{I}_{\text {pre }}$ ) associated to the ZmSUT1 activities were gained by subtracting the current transients at different voltages in the presence and absence of sucrose (Fig. 3B). The integral of the presteady-state currents over time plotted against the voltage reflect the associated charge-voltage curve (Fig. 3G). Note, that there is no significant difference from the "on" (integral of $\mathrm{I}_{\text {pre }}$ during the application of the voltage pulse) and the "off" charge (integral of $\mathrm{I}_{\text {pre }}$ during repolarisation; Fig. 3D).

When fitting $Q$ vs $\mathrm{V}$ with the Boltzmann equation (continuous line in Fig. 3C, see materials and methods) a half activation voltage of $-59 \pm 4 \mathrm{mV}$ (mean \pm SEM, $\mathrm{n}=20$ ) was calculated. Using the derivative of the charge over time the capacitance change associated with the presteady-state current, was obtained (Fig. 3C inset, open circles). Note, that the calculated capacitance changes were well in agreement with the experimentally determined capacitance (black line) using small voltage steps according to the methods developed by Adrian and Almers [28] (see materials and methods, Fig. 3D). The good agreement between these two quantities guarantees that the mentioned experimental approach does not harbor significant problems of voltage-clamp due to the large potential pulses ( $\Delta \mathrm{V}$ up to $100 \mathrm{mV}$ ). The slope of the Boltzmann fit of $98 \pm 3 \mathrm{mV}$ (mean $\pm \mathrm{SEM}, \mathrm{n}=20$ ) is equal to KT/ ez $\delta$ where $\mathrm{K}$ and $\mathrm{T}$ have the usual meaning, e is the elementary charge, $\mathrm{z}$ the valence of the moving charge and $\delta$ the fraction of the membrane field through which the charge moves; therefore $z \delta$ is equal to 0.26 . If the moving charge is monovalent, then it moves $26 \%$ of the electrical distance from the external solution to the cytoplasmic side of the membrane. The decay of the presteadystate currents could be described by two exponential functions (inset of Fig. 3E). The exponential fitting identified two reliable time constants (Fig. 3E, see Materials and Methods). The fast component of less than $1 \mathrm{~ms}$ is biased by the speed of the clamp of the amplifier (squares). The slow time constant was around $2 \mathrm{~ms}$ at negative voltages and up to $3 \mathrm{~ms}$ at positive voltages (circles). The cross symbols in Fig. 3E represent the slow time constants derived from the exponential fitting of $\mathrm{I}_{\text {pre }}$ "off". Their averages served as an estimate of the time constant of the "on" presteady-state current at the holding voltage of $-20 \mathrm{mV}$.

\section{Relationship between $\mathrm{I}_{\text {pre }}$ and $\mathrm{I}_{\mathrm{tr}}$}

The transport-associated currents in the presence of external sucrose were apparently different to the presteady-state currents obtained in the absence of sucrose. The gating currents of voltage dependent ion channels appear independent from the presence or absence of the permeating ion [29]. Therefore these gating charges and the transport-associated currents are clearly distinct and not predictable. To test whether there exists a relation between $\mathrm{I}_{\text {pre }}$ in the absence of sucrose and $\mathrm{I}_{\mathrm{tr}}$ in the presence of sucrose with ZmSUT1, we used the relationship $I=Q / \tau$ to predict the transport-associated currents solely from the $I_{\text {pre }}$ parameters 

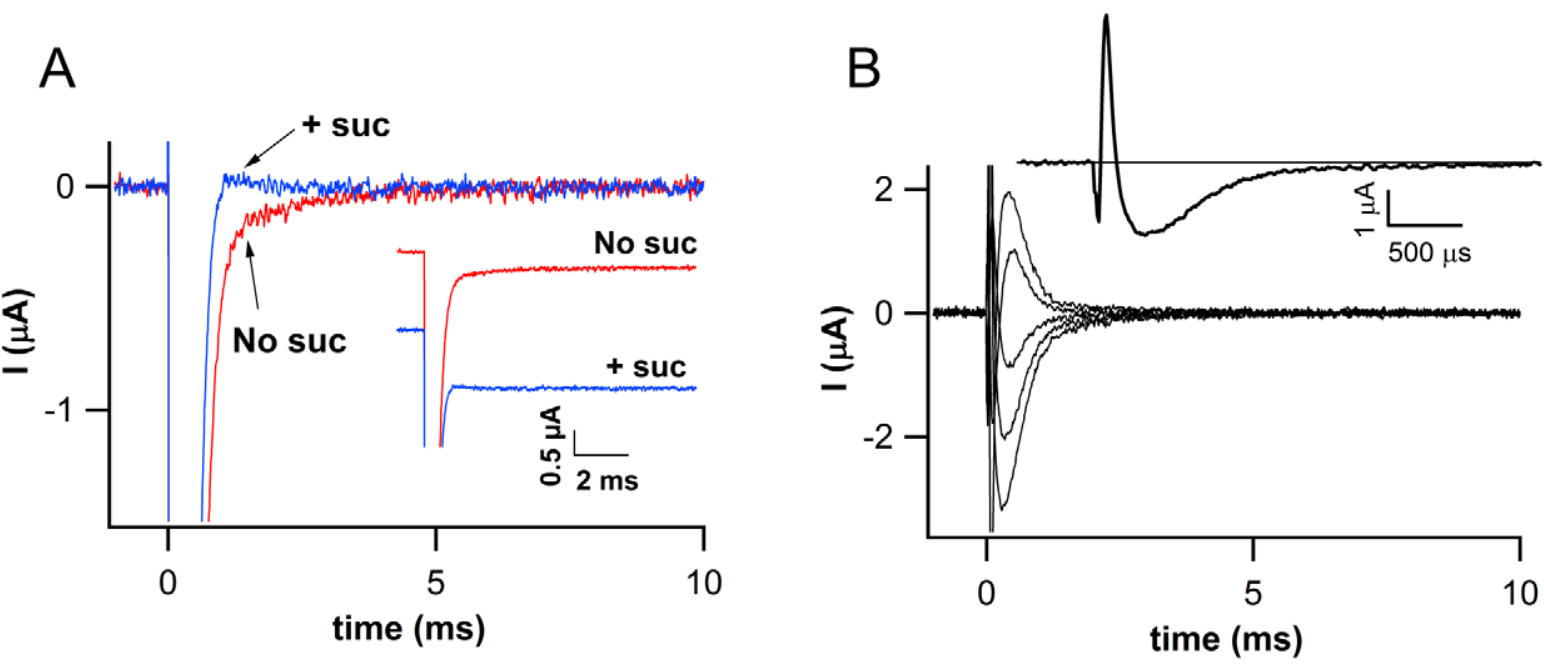

C
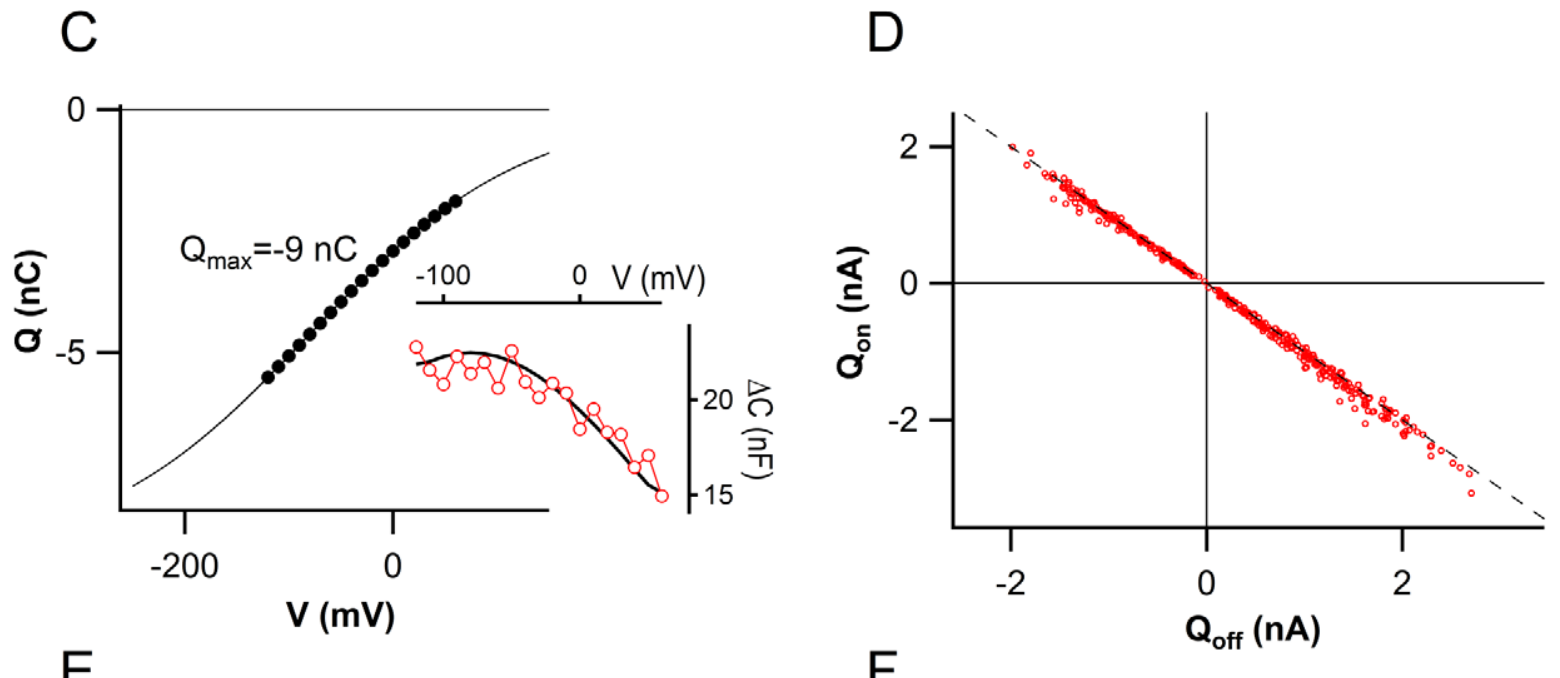

$\mathrm{E}$

$\mathrm{F}$
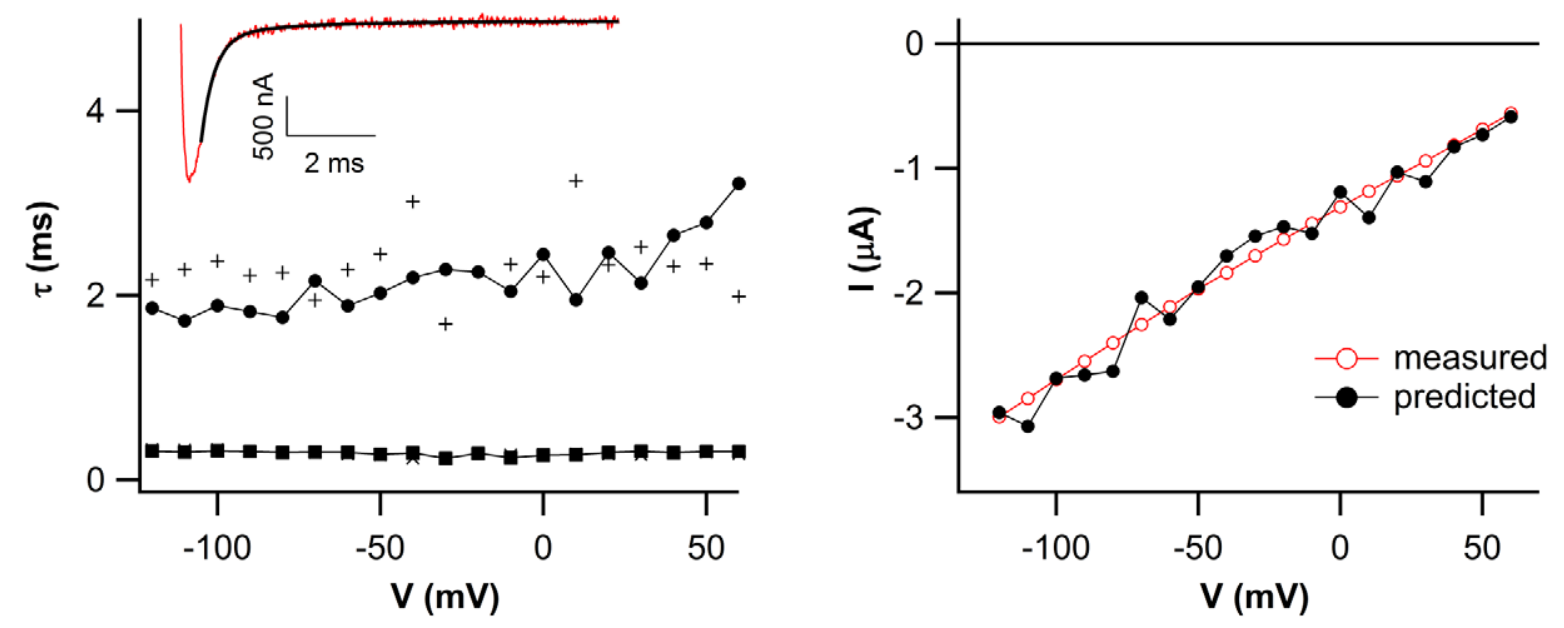

Figure 3. Presteady-state currents of ZmSUT1. A) Inset: currents recorded in the absence (No suc) and in the presence of saturating sucrose concentration (+suc). Same currents presented in the inset after forcing their stationary level to zero. Holding and pulse voltages were -20 and $-80 \mathrm{mV}$ respectively. B) Presteady-state currents versus time obtained after subtracting the stationary currents in the absence and in the presence of saturating sucrose. Holding voltage $-20 \mathrm{mV}$, voltage pulses from $+40 \mathrm{mV}$ to $-120 \mathrm{mV}$ (step $-40 \mathrm{mV}$ ). Inset: presteady-state current elicited by a voltage of $-80 \mathrm{mV}$. C) Charge associated to presteady-state currents plotted versus the voltage. The charge was obtained from integration of the presteady-state currents (see Materials and Methods). The continuous line is the fit of $\mathrm{Q}$ with the Boltzmann function shown in Material and Methods with, in this oocyte, $Q_{\max }=-9 \mathrm{nC}, \mathrm{V}_{\mathrm{h}}=-80 \mathrm{mV}$ and $\mathrm{s}=103 \mathrm{mV}$. Inset of panel C: Capacitance (empty symbols) measured using the method of Adrian and Almers (1976). The continuous line is the capacitance derived from the Q-V plot of panel C (see materials and methods). D) $Q_{o n}$ and $Q_{o f f}$ the charge movements during the on and off response of the voltage steps obtained from the integrals of the presteady-state currents in 20 different 
oocytes, are plotted for various membrane voltages from -120 and $+60 \mathrm{mV}$ in $10 \mathrm{mV}$ steps. The dotted line has a slope of 1 . The linear fits (not shown) of the experimental points for each single oocyte give regression coefficients with a minimum value of -0.993 , indicating very strong correlation. E) Time constants of the decay of the on and off presteady-state currents versus the applied voltage. The final decay of the presteadystate currents can be fitted by a two-exponential function with a fast and a slow $\tau$ (see Materials and Methods). The value of the fast time constants (- for the on and for the off presteady-state current) is under $1 \mathrm{~ms}$ and is limited by the speed of the voltage-clamp. Symbols $\bullet$ and + represent the slow time constants of the on and off presteady-state currents respectively. F) Transported (measured, empty symbol) and predicted (filled symbol) currents plotted against voltage for a single oocyte.

doi:10.1371/journal.pone.0012605.g003

obtained without sucrose (cf. [30,31]). Indeed we could obtain a very good agreement between the measured transport-associated currents (open circles) and predicted currents (filled symbols, Fig. 3F) indicating that there is an inter-relation between $\mathrm{I}_{\text {pre }}$ and $\mathrm{I}_{\mathrm{tr}}$.

\section{ZmSUT1 Turns Over 500 Sucrose Molecules per Second}

In Fig. $4 \mathrm{~A}$ we plotted the transport-associated currents $\left(\mathrm{I}_{\mathrm{tr}}\right)$, recorded at $-120 \mathrm{mV}$ in 20 oocytes differing in their individual level of ZmSUT1 expression, against the corresponding charges $\left(Q_{-120}\right)$. The linear fit showed a clear correlation between the two quantities (regression coefficient of 0.92), indicating that both $\mathrm{I}_{\mathrm{tr}}$ and $Q$ depend on the total number of transporters present on the membrane of the oocyte. Interestingly, and as expected from our previous analysis (Fig. 3F), the slope of the fit was $520 \mathrm{~s}^{-1}$ very close to the reciprocal of the slow time constant shown in Fig. 3E (about $500 \mathrm{~s}^{-1}$ ). The transport-associated currents of ZmSUT1expressing oocytes with different levels of expression in Fig 4B were obtained by the subtraction of the currents in the absence from the currents in the presence of saturating sucrose concentration (open symbols). The filled symbols were again calculated using the ratio $Q / \tau$ based only on parameters obtained from the presteady-state currents in the absence of sucrose. The good agreement between the measured and predicted currents confirms that the prediction of $\mathrm{I}_{\mathrm{tr}}$ from $\mathrm{I}_{\text {pre }}$ is independent from the expression level of $\mathrm{ZmSUT1}$. In this respect, the turnover rate of the transporter is about $500 \mathrm{~s}^{-1}$ at negative (physiological) voltages which was calculated from the reciprocal of the slow time constant (see Fig. 3E) or from the slope of the linear fitting of I. $120 \mathrm{mV}$ vs Q $120 \mathrm{mV}$ (Fig. 4B). Moreover we can estimate the average number of transporters from the equation: $Q_{\max }=\mathrm{Nez} \delta$ where $\mathrm{Q}_{\max }$ is the total charge movement, $\mathrm{N}$ the number of transporter per oocyte, e the elementary charge and $z \delta$ was obtained by the Boltzmann analysis performed above. Considering a mean $Q_{\max }$ of $8 \mathrm{nC}, \mathrm{N}$ is $2 \times 10^{11}$ transporter molecules per oocytes, a value well in line with other transporters (i.e. GAT1 [15], KAAT1 [14]) confirming the capability of the oocyte system to express foreign proteins at very high densities. In our experiments the average capacitance of oocytes was $170 \mathrm{nF}$. Using the specific capacitance of $1 \mu \mathrm{F} / \mathrm{cm}^{2}$ of biological membranes we obtain an average oocyte area of $1.7 \times 10^{7} \mu \mathrm{m}^{2}$ and a transporter density of about $10^{4} / \mu \mathrm{m}^{2}$. If each transporter effectively covers an approximate area of $5 \mathrm{~nm}^{2}$ (Abramson, 2003), ZmSUT1 would cover about 25\% of the total oocyte area.

\section{Sucrose Affects ZmSUT1-Derived Presteady-State Currents}

The presteady-state currents of $\mathrm{ZmSUT1}$, obtained in a similar manner as those shown in Fig. 3, appeared modulated by the external sucrose concentration. Application of 0.5 and $1 \mathrm{mM}$ external sucrose resulted in a sucrose-dependent reduction of $\mathrm{I}_{\text {pre }}$ (Fig. 5A). The charge associated to $\mathrm{I}_{\text {pre }}$ versus voltage decreased upon increasing the external sucrose concentration (Fig. 5B) and its derivative was in agreement with the measured capacitance (Fig. S1A). There was, however, no significant effect of external sucrose at concentration equal to 0.5 and $1 \mathrm{mM}$ on the slow time constant (Fig. 5C). The relationship $\mathrm{I}=\mathrm{Q} / \tau$ previously used to predict the transport currents solely from the knowledge of the presteady-state currents can be written as:

$$
I_{\text {sat }}-I_{0}=\left(Q_{0}-Q_{\text {sat }}\right) / \tau
$$

where $I_{\text {sat }}$ and $I_{0}$ represent the transport-associated currents in the presence and absence of saturating sucrose, $Q_{\text {nosuc }}$ and $Q_{\text {sat }}$ are the charges associated to the transient component of the transporter current in the absence and presence of the sugar and $\tau$ represents the slow time constant of the exponential fitting of $\mathrm{I}_{\text {pre }}$. Equation 1 can be extended to predict the ZmSUT1 response to

A

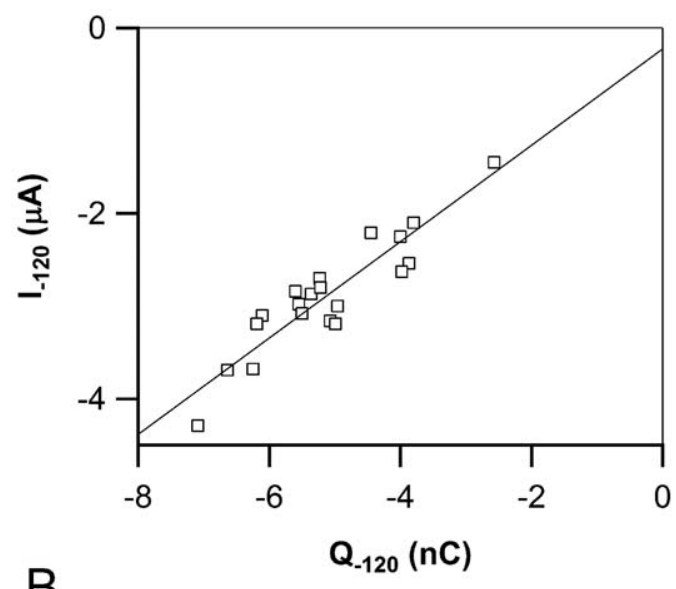

B

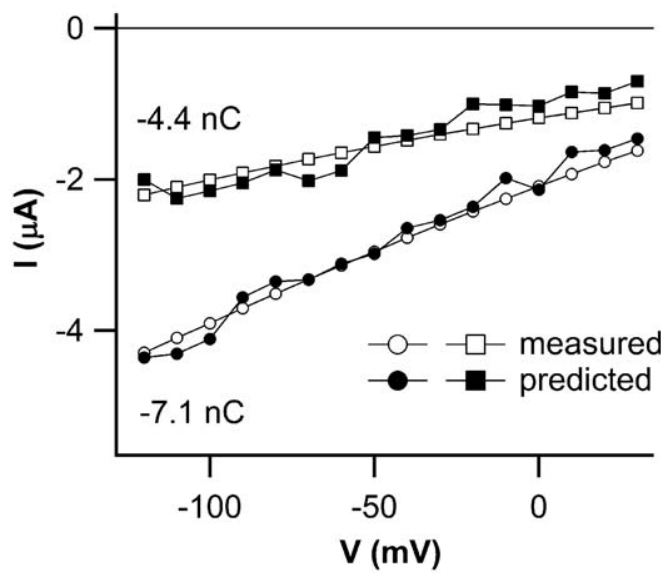

Figure 4. Properties of charge movement. A) Relation between $\mathrm{I}_{-120}$ and $\mathrm{Q}_{-120}$ for 20 individual oocytes; the linear regression $(r=0.92)$ gives a slope of $520 \pm 53 \mathrm{nA} \mathrm{nC}$ or s$^{-1}$. C) Transported (measured, empty symbols) and predicted (filled symbols) currents plotted against voltage for two oocytes with different level of ZmSUT1 expression. doi:10.1371/journal.pone.0012605.g004 
given external sucrose concentration $(\mathrm{X})$ :

$$
I_{\text {sat }}-I_{X}=\left(Q_{X}-Q_{\text {sat }}\right) / \tau
$$

where $I_{X}$ and $Q_{X}$ are respectively the stationary current and the charge associated to the transient component of the ZmSUT1 current in the presence of a sucrose concentration equal to $\mathrm{X}$. To compare the measured and the predicted transport currents the $I_{\text {sat }}-I_{X}$ values, obtained from the transport-associated currents, were normalized (see Materials and Methods) and plotted as a function of voltage (open symbols, Fig. 5C). The filled symbols were calculated using equation 2 with parameters obtained from the experimentally recorded $\mathrm{I}_{\text {pre }}$ (for the normalization procedure see Materials and Methods). Please note the good agreement between the experimental and predicted data over a broad voltage range. This agreement strongly suggests a similar origin of the presteady-state currents and the transport-associated currents. One might thus conclude that $\mathrm{I}_{\text {pre }}$ in the absence of sucrose reflect proton movements from an external to an inner position of the transporter and that $\mathrm{I}_{\mathrm{tr}}$ reflects the proton permeation across the membrane when sucrose is added [7].

\section{Protons Affect the Gating of ZmSUT1}

When the proton concentration was reduced from $\mathrm{pH} 4$ to $\mathrm{pH} 7$ a strong reduction of $\mathrm{I}_{\text {pre }}$ was observed (Fig. 6A). To gain the voltage dependence of this process we plotted the $\mathrm{I}_{\text {pre }}$-associated charge $\mathrm{Q}$ in different external $\mathrm{pH}$ buffers against the applied voltage (Fig. 6B). Plotting the half activation voltage, obtained in panel B, against decreasing external proton concentration (Fig. 6C) visualized the shift of $\mathrm{Q}(\mathrm{V})$ towards negative voltages. A linear fit from $\mathrm{pH} 7$ to $\mathrm{pH} 4$ resulted in a slope of $83 \pm 5 \mathrm{mV}$ per $\mathrm{pH}$ unit $(n=4)$. These results are in agreement with our notion that protons generate ZmSUT1 presteady-state currents. As expected, the derivative of the charge is in line with the actually measured capacitance as shown in Fig. S1. The slow time constants of $\mathrm{I}_{\text {pre }}$ at pH 5 and 4 are not significantly different (Fig. 5D). In line with our experiments at $\mathrm{pH} 4$, the agreement between the predicted and measured stationary currents at $\mathrm{pH} 5$ were satisfactory (Fig. 6, see Materials and Methods for details).

\section{Discussion}

The capacitance $\left(\mathrm{C}_{\mathrm{m}}\right)$ of biological membranes represents a basic electrical property. Using the paired ramp technique [26] we could in real-time monitor changes in $\mathrm{C}_{\mathrm{m}}$ of Xenopus oocytes over a broad range of physiological conditions such as $\mathrm{pH}$-, sucrose- and membrane potentials changes. The robustness of the $\mathrm{C}_{\mathrm{m}}$ measurement technique allowed us to measure $\mathrm{C}_{\mathrm{m}}$ even under conditions where presteady-state measurements were biased by the speed of the voltage-clamp and therefore beyond the resolution limit of the method.

The Wright lab in 1996 recorded presteady-state currents of the potato $\mathrm{H}^{+}$/sucrose cotrasporter StSUT1 [8]. In contrast to ZmSUT1 those of StSUT1 appeared sucrose independent. In the presence of saturating extracellular sucrose steady state currents increased without affecting the transient component. Compared to water-injected oocytes, StSUT1 expressing ones exhibited a large, $\mathrm{pH}$-dependent transient component. Acidification of the extracellular solution did not change both the total charge $\left(Q_{\max }\right)$ and the apparent valence $(\mathrm{z})$ but shifted the Boltzmann curve $30 \mathrm{mV}$ more negative. Therefore it was not possible with the weak StSUT1 activity in oocytes to correlate the effect of the membrane voltage and the $\mathrm{H}^{+}$concentration on presteady-state kinetics with those of transport currents (steady- state). How can this discrepancy to the situation with ZmSUT1 be explained? The transient components in StSUT1 could reflect structural arrangements of the carrier protein. With ZmSUT1 expressing oocytes, sucrose-independent presteady-state currents were not apparent. The transient component in the presence of saturating sucrose was rather comparable to those of water injected oocytes. Stationary currents with StSUT1 injected oocytes were 20-50 times smaller than those obtained with ZmSUT1. The discrepancy between Zea maize and Solanum tuberosum transporter may thus reflect a problem of resolution only.

Taking advantage of the high transport activity of ZmSUT1, we could measure presteady-state currents with a shift of the associated charge by about $80 \mathrm{mV}$ upon acidification. The $\mathrm{I}_{\text {pre }}$ voltage-dependence of $\mathrm{ZmSUT1}$ is in agreement with the range of physiological membrane potential of the plant cell. E.g. at an extracellular $\mathrm{pH}$ of $5-5.5$, the half-activation voltage $\mathrm{V}_{1 / 2}$ of $\mathrm{I}_{\mathrm{pre}}$ is more negative than $-120 \mathrm{mV}$. Since ZmSUT1 is capable to mediate sucrose loading as well as unloading [7], the slope of about $100 \mathrm{mV}$ indicates that the $\mathrm{H}^{+}$/sucrose cotransporter might indeed operate in a wide voltage range.

The experimental analysis of the sugar and proton dependent ionic currents associated with the gating and solute movement of ZmSUT1 point to the following transport mechanism: the proton entrance into the transporter is far from the electric field (indicated by the voltage-independence of $\tau$ ) and was the rate limiting step with respect to the overall transport mechanism under our specific experimental conditions. It is worth noting that this rate limiting step is not necessarily caused by the diffusion and/or binding of the protons inside ZmSUT1 but could be influenced by the accessibility of the proton binding site due to conformational changes of the transporter. As proposed by Kaback's lab (e.g. [32,33]) for ioncoupled cotransporters, in the absence of substrate the transporter can have two main conformations for the proton binding site, respectively facing the extracellular (outward-facing) and the cytosolic side (inward-facing). The slow time constant measured in this work could represent the transition between these two states. In the absence of sugar the proton is trapped inside the transporter and can move up to $25 \%$ of the electrical distance across the membrane. This voltage-dependent movement is the origin of the presteadystate currents. In this case the transporter can be seen as a pure capacitor and the protons are the relative charges. When saturating sucrose concentrations are present at the extracellular surface of ZmSUT1, the proton inside the transporter is released into the cytoplasm from the inward-facing conformation. When crossing the barrier one proton moves one sugar molecule into the cytoplasm [7]. Very likely this step is associated with a conformational change of the transporter [11,32]. From our data we predict that the conformational change that moves the protons from the extracellular to the cytosolic side in the presence of sucrose has to be faster than $2 \mathrm{~ms}$. In this scenario the transporter can be seen as a pure resistor and the protons are the charges giving rise to the electrical transport current. Under conditions of limiting sugar in the extracellular milieu the transporter behaves like a capacitor as well as a resistor. Furthermore the relationship between presteady-state and transport-associated currents suggests that the proton entry into the electric field across ZmSUT1 is not influenced by the presence of external sucrose. This link points to a common origin of the presteady-state and the transport-associated currents, which is underpinned by the fact that the reduction of the external $\mathrm{H}^{+}$ concentration decreased both the presteady-state and the transportassociated currents.

Transporters both from animal (SGLT1, PepT1, [24,34]) and plant kingdoms (STP1, [35]) are characterized by presteady-state currents which are apparent in the absence of external substrate 

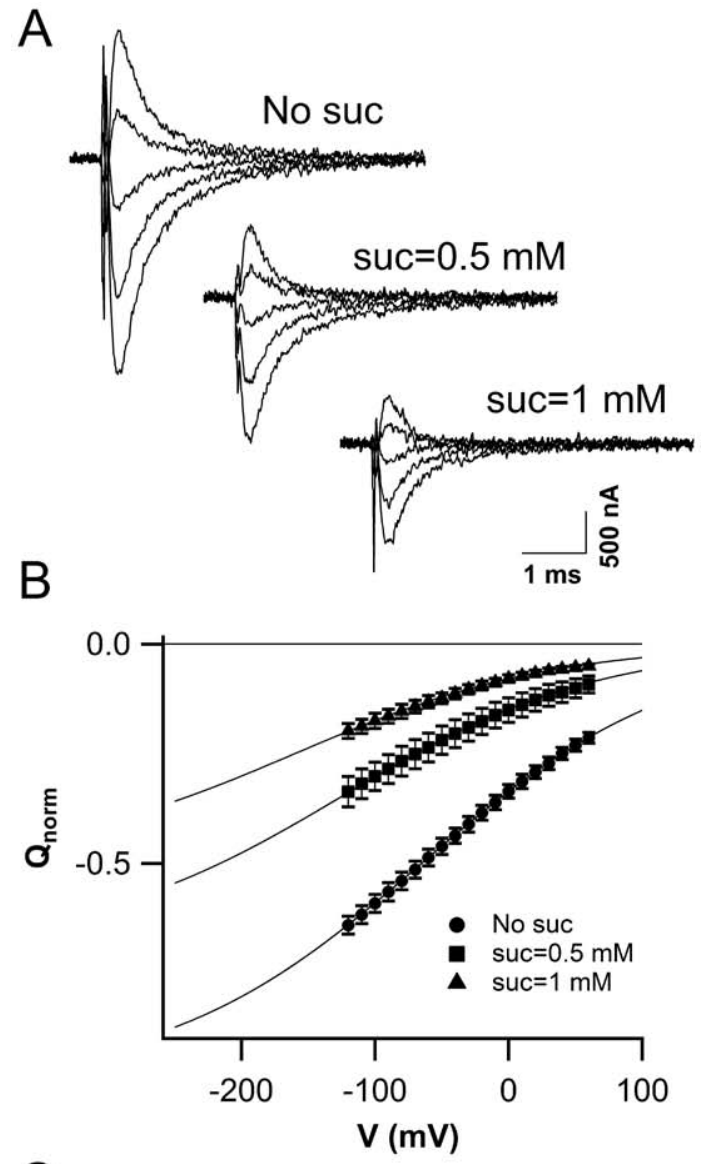

C
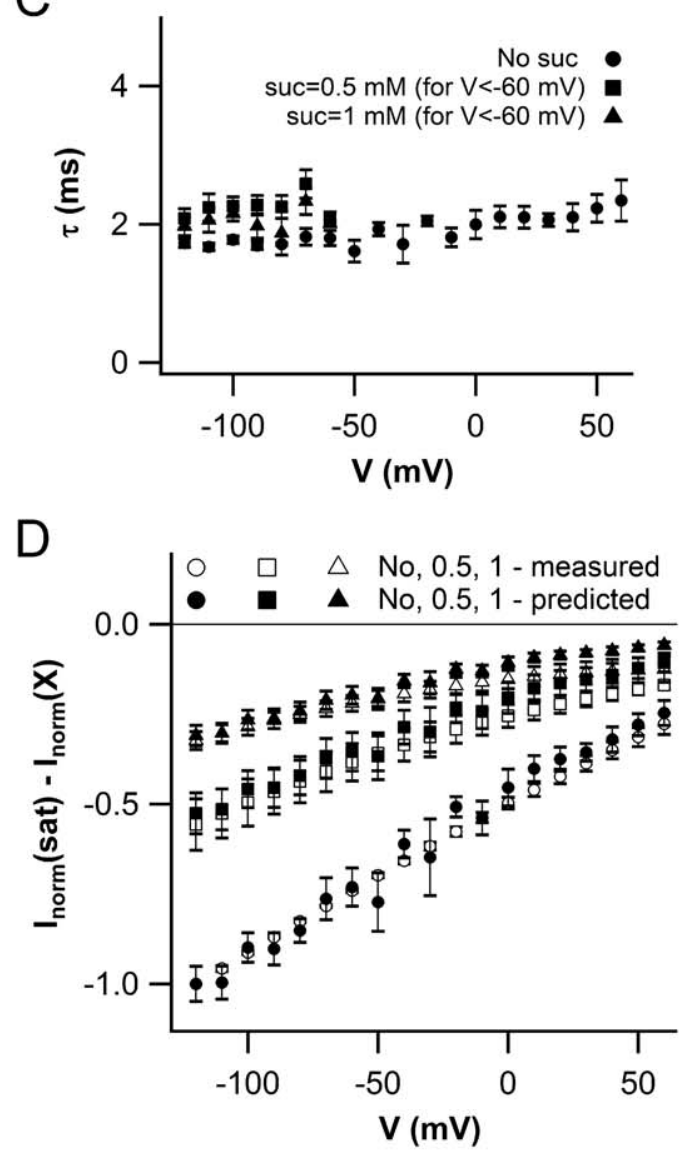

Figure 5. Sucrose dependence of ZmSUT1 presteady-state currents. A) Presteady-state currents of a single oocyte at varying external sucrose concentrations. For the sake of clarity only currents recorded from +40 to $-120 \mathrm{mV}$, in $40 \mathrm{mV}$ step, are shown; the holding voltage was $-20 \mathrm{mV}$. B) Charge associated to presteady-state currents versus voltage at different sucrose concentrations. The continuous lines are fit of Q using a Boltzmann function (see Materials and Methods). Data for each oocyte were normalized to the maximum charge obtained in the absence of external sucrose, $Q_{\max }$ (No suc). The fits gave the following parameters (mean $\pm S E M, n=4$ ): no external sucrose: $\mathrm{V}_{1 / 2}=-65 \pm 8 \mathrm{mV}, \mathrm{s}=96 \pm 5 \mathrm{mV} ; 0.5 \mathrm{mM}$ sucrose: $\mathrm{V}_{1 / 2}=-123 \pm 17 \mathrm{mV}$, $\mathrm{Q}_{\max }($ suc $=0.5 \mathrm{mM}) / \mathrm{Q}_{\max }($ No suc $)=0.69 \pm 0.02 ; 1 \mathrm{mM}$ sucrose $\mathrm{V}_{1 / 2}=-159 \pm 20 \mathrm{mV}, \mathrm{Q}_{\max }(\mathrm{suc}=1 \mathrm{mM}) / \mathrm{Q}_{\max }$ (No suc) $=0.50 \pm 0.02$. At 0.5 and $1 \mathrm{mM}$ sucrose the slope $\mathrm{s}$ of the fit was fixed at the value obtained at 0 external sucrose. C) $I_{\text {sat }}-I_{X}$ against the applied membrane potential. $I_{\text {sat }}$ is the current at saturating external sucrose concentration; $I_{x}$ are the currents in the presence of $0,0.5 \mathrm{mM}$ and $1 \mathrm{mM}$ external sucrose. Empty and filled symbols refer to the measured and predicted currents respectively. The following relationship was used in order to evaluate the predicted currents: $I_{\text {sat }}-I_{X}=Q_{X} / \tau$ where $X$ represents the external sucrose concentration and $\tau$ is the slow time constant at zero external sucrose.

doi:10.1371/journal.pone.0012605.g005

and disappear under substrate saturation. In GAT1 and GlyT1 transporters (respectively a sodium/chloride GABA and glycine cotransporter present in synapses of animal cells) a possible interconversion between the two kinds of currents was recently explored $[30,31,36]$. Presteady-state currents of GAT1 and GlyT1 result from the displacement of $\mathrm{Na}^{+}$or chloride ions within the electrical field of the membrane traveling between the extracellular space and a binding site in the protein. Interestingly Fesce et al. (2002, [30]) and Cherubino et al. (2010, [36]) could predict transport-associated currents in the presence of GABA or glycine by analyzing presteady-state currents in the absence of substrate. For these animal cotransporters it was proposed that in the absence of substrate sodium/chloride could move, with a ratelimiting speed represented by $\tau$, from an external to an internal site of the transporter generating the presteady-state currents. Upon addition of substrate the 'trapped' ion is able to cross the membrane with the same rate-limiting step. Our analyses with ZmSUT1 point to a similar relationship between the transient current component and the transport-associated currents. Future studies will thus need to focus on the question whether similar relationships between charge movement and transport-associated currents found in different cotransporter types have common grounds.

\section{Materials and Methods}

An ethics statement is not required for this work.

\section{TEVC analysis in Xenopus Oocytes}

ZmSUT1 cRNA was prepared using the mMESSAGE mMACHINE $^{\text {TM }}$ RNA Transcription kit (Ambion Inc., Texas, USA). Oocyte preparation and cRNA injection have been described elsewhere [37]. In two-electrode voltage-clamp studies oocytes were perfused with a standard-solution containing $30 \mathrm{mM}$ $\mathrm{KCl}, 1 \mathrm{mM} \mathrm{CaCl}{ }_{2}$, and $1.5 \mathrm{mM} \mathrm{MgCl}_{2}$ based on Tris/Mes buffers for $\mathrm{pH}$ values from 5.5 to 7.5 or based on citrate/Tris buffers for the $\mathrm{pH}$ values 3.5 to 5.0. Solutions were adjusted to 220 mosmol kg ${ }^{-1}$ using D-sorbitol.

\section{Transport Current and Membrane Capacitance Measurements}

Simultaneous measurements of transport currents $\left(\mathrm{I}_{\mathrm{tr}}\right)$ and membrane capacitance $\left(\mathrm{C}_{\mathrm{m}}\right)$ were performed using an TEC-10X 
A
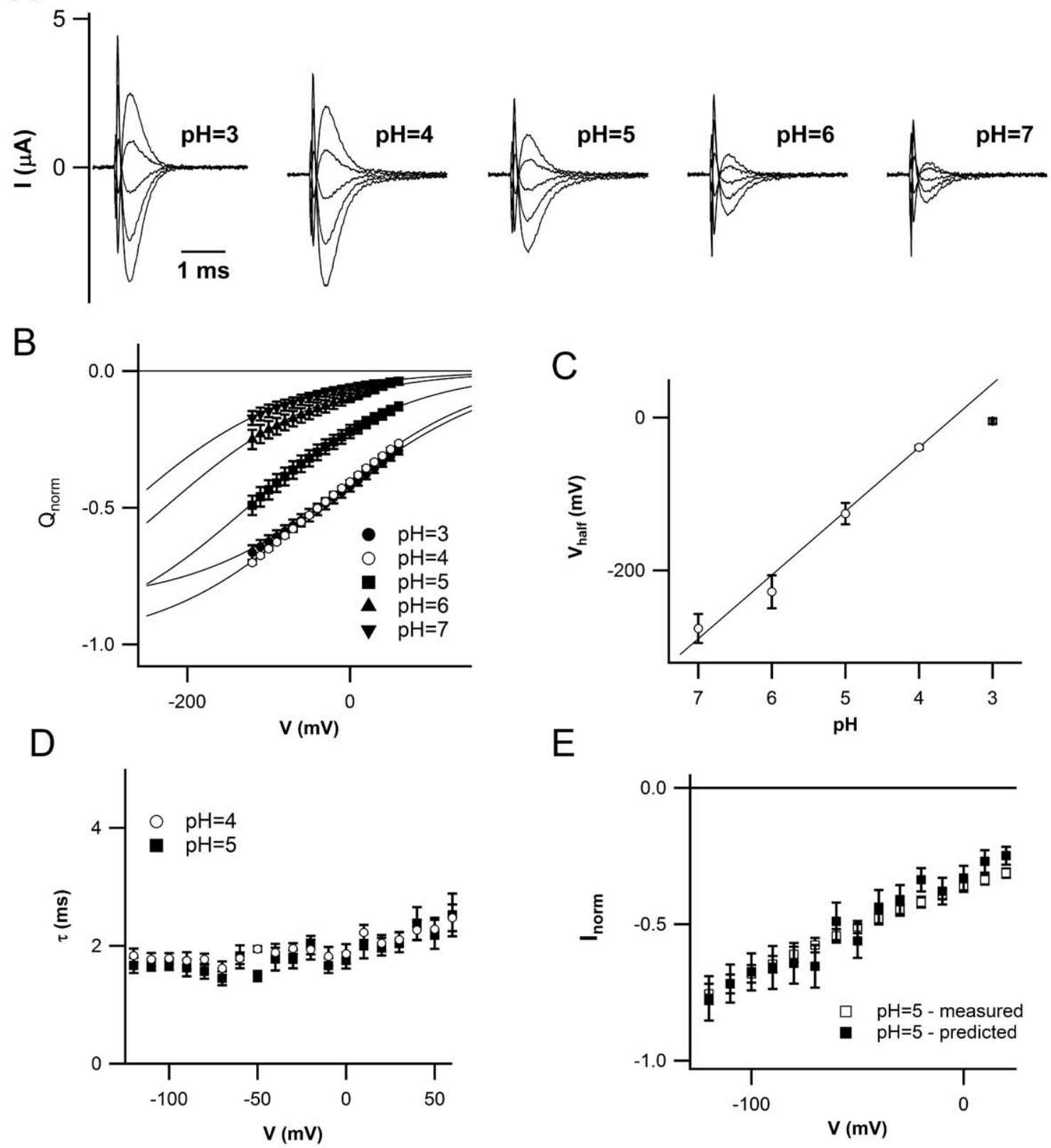

$\mathrm{E}$

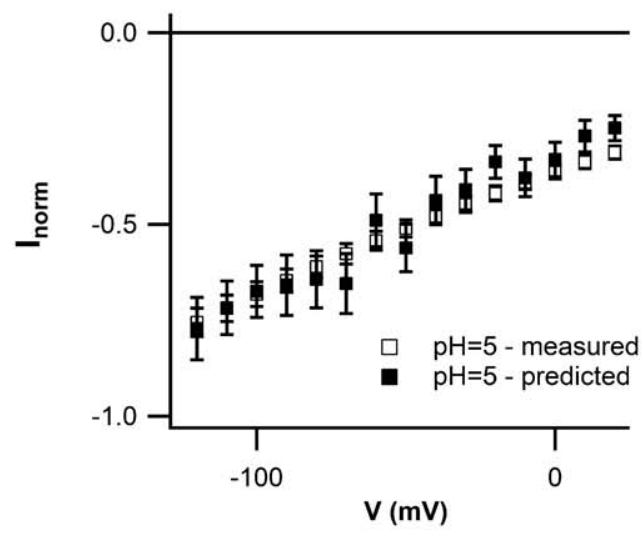

Figure 6. pH dependence of ZmSUT1 presteady-state currents. A) Presteady-state currents of a single oocyte at varying external pH. Holding voltage $-20 \mathrm{mV}$, voltage pulses from $+40 \mathrm{mV}$ to $-120 \mathrm{mV}$ (step $-40 \mathrm{mV}$ ). B) Normalized charge associated to presteady-state currents at different external $\mathrm{pH}$ versus voltage. For the details of the $\mathrm{pH}$ analysis see Materials and Methods. $\mathrm{Q}_{\max }(\mathrm{pH}=3) / \mathrm{Q}_{\max }(\mathrm{pH}=4)=0.85 \pm 0.03$. Data were obtained by four different oocytes. C) Half activation voltages $\left(\mathrm{V}_{1 / 2}\right)$ obtained by data in panel $\mathrm{B}$ versus external $\mathrm{pH}$. The slope of the linear fit from $\mathrm{pH} 7$ to 4 is equal to $83 \pm 5 \mathrm{mV}$. D) Slow time constant $\tau$ recorded at $\mathrm{pH} 4$ and 5 plotted against applied voltage. E) Predicted (filled symbols) and measured (empty symbols) transported currents versus voltage (see Materials and Methods for details).

doi:10.1371/journal.pone.0012605.g006

amplifier (NPI Electronic, Tamm, Germany) controlled by the PULSE and X-Chart software (HEKA Electronics). $\mathrm{C}_{\mathrm{m}}$ was measured using a previously described paired ramps approach [26]. Sucrose-induced proton currents and sucrose-induced capacitance changes were recorded at $-20 \mathrm{mV}$ while the oocytes were super-fused with different external solutions. When the bath medium was completely exchanged $\mathrm{C}_{\mathrm{m}}$ and $\mathrm{I}_{\mathrm{tr}}$ were determined in the voltage range between +20 and $-120 \mathrm{mV}$ in $10 \mathrm{mV}$ decrements.

Alternatively we use the method developed by Adrian and Almers (see also [14]): a small voltage step of $20 \mathrm{mV}(\Delta \mathrm{V})$ and duration of $20 \mathrm{~ms}$ was superimposed to a given applied voltage. The amount of moved charge $\Delta \mathrm{Q}$ was obtained by integrating the current transient. The slope of the membrane capacitance at the applied voltage was approximated by the ratio $\Delta \mathrm{Q} / \Delta \mathrm{V}$. We verified that the two methods of measuring membrane capacitance described above gave similar results (data not shown).

\section{Presteady-State Currents}

Presteady-state currents were obtained using the two-electrode voltage-clamp technique (TECV) subtracting the traces at 
saturating sucrose concentration (typically $100 \mathrm{mM}$ ) and forcing the steady level at the end of each voltage pulse to zero. The holding potential was maintained at $-20 \mathrm{mV}$ and voltage pulses of $20 \mathrm{~ms}$ from $60 \mathrm{mV}$ to $-120 \mathrm{mV}$ in $-10 \mathrm{mV}$ steps were applied. Signals were sampled at $100 \mathrm{kHz}$ and filtered at $20 \mathrm{kHz}$.

The charge $(\mathrm{Q})$ associated to each presteady-state currents was obtained using the following procedure: a) the charge $Q_{\exp }$ was calculated by the integral of the presteady-state currents and fitted by the Boltzmann equation $\mathrm{Q}_{\max } /\left(1+\exp \left[\left(\mathrm{V}-\mathrm{V}_{1 / 2}\right) / \mathrm{s}\right]\right)+\mathrm{Q}_{\mathrm{i}}$, where $Q_{i}$ represents the charge offset, $Q_{\max }$ the maximum charge, $\mathrm{V}_{1 / 2}$ the half-activation voltage and $\mathrm{s}$ is linked to the slope of the Boltzmann curve at $V=V_{1 / 2}$. The charge $Q$ was $Q_{\exp }-Q_{j}$. The "on" charge, the charge calculated when the voltage pulse was applied, and the "off" charge, calculated when the potential was again set to the holding value, did not differ considerably (see Fig. 3D).

The last part of the decay of the presteady-state currents was fitted by the two exponential function $I_{\text {fast }} \exp \left(-t / \tau_{\text {fast }}\right)+I_{\text {slow }}$ $\exp \left(-t / \tau_{\text {slow }}\right)$. In order to verify the reliabilty of $\tau$, the starting time of the fit was increased in step of $50 \mu \mathrm{s}$; in each step the fitting procedure was applied (up to 10 steps) and $\tau$ was calculated as the average value. When the relative error (SD/mean) of $\tau$ was greater that $10 \%$ the point was discarted; this was the case for sucrose concentrations equal to 0.5 and $1 \mathrm{mM}$ at voltages more positive than $-60 \mathrm{mV}$ and external $\mathrm{pH}$ of 7, 6 and 3 (see Fig. 5 and 6).

To obtain $\tau$ at the holding voltage $\left(\mathrm{V}_{\text {hold }}\right)$ a similar fitting procedure was performed on the off charge at voltages different from $V_{\text {hold }} \tau\left(V_{\text {hold }}\right)$ was calculated as the average value of $\tau_{\text {off }}(V)$. The transported currents induced by sucrose were calculated as the difference of the stationary currents in the presence and the absence of sucrose respectively. The predicted current was obtained using the following relationship: $I=Q / \tau$. Changes in capacitance associated with the presteady-state currents $\left(\mathrm{C}_{\mathrm{p}}\right)$ were evaluated as the derivative of $\mathrm{Q}$ with respect to $\mathrm{V}$ and compared with the measured capacitance. Only the experiments with $\mathrm{C}_{\text {pred }} \sim \mathrm{C}_{\mathrm{m}}$ were considered.

Analysis of the sucrose dependency. For the analysis of the sucrose dependency shown in Fig. 5 presteady-state currents at 0 , 0.5 and $1 \mathrm{mM}$ sucrose were obtained from the experimental currents subtracting the traces at saturating sucrose concentration and forcing the steady level to zero at the end of each voltage pulse. The integral of the presteady-state currents from a single oocyte was fitted by the Boltzmann equation shown above. At 0.5 and $1 \mathrm{mM}$ sucrose the slope $\mathrm{s}$ of the fit was fixed at the value obtained at $0 \mathrm{mM}$ external sucrose. $\mathrm{Q}_{\mathrm{X}}$ was calculated as $\left(\mathrm{Q}_{\exp }(\mathrm{X})\right.$ $\left.\mathrm{Q}_{\mathrm{i}}(\mathrm{X})\right) / \mathrm{Q}_{\max }(0)$ with $\mathrm{X}$ equal to $0,0.5$ and $1 \mathrm{mM}$ external sucrose. The predicted currents were calculated using equation 2 (see Results session) with the slow time constant obtained in the absence of external sucrose; as a matter of fact $\tau$ was not significantly affected by external sucrose concentrations of 0.5 and $1 \mathrm{mM}$ (see

\section{References}

1. Van Bel AJE (2003) The phloem, a miracle of ingenuity. Plant, Cell \& Environment 26: 125-149.

2. Sauer N (2007) Molecular physiology of higher plant sucrose transporters. FEBS Letters 581: 2309-2317.

3. Aoki N, Hirose T, Takahashi S, Ono K, Ishimaru K, et al. (1999) Molecular cloning and expression analysis of a gene for a sucrose transporter in maize ( $\mathrm{eea}$ mays L.). Plant Cell Physiol 40: 1072-1078.

4. Riesmeier JW, Hirner B, Frommer WB (1993) Potato sucrose transporter expression in minor veins indicates a role in phloem loading. Plant Cell 5: 1591-1598.

5. Sauer N, Stolz J (1994) SUC1 and SUC2: two sucrose transporters from Arabidopsis thaliana; expression and characterization in baker's yeast and identification of the histidine-tagged protein. Plant J 6: 67-77.

6. Slewinski TL, Meeley R, Braun DM (2009) Sucrose transporterl functions in phloem loading in maize leaves. J Exp Bot 60: 881-892.
Fig. 5C). The predicted and measured currents for a single oocyte were normalized at the value of $\left(\mathrm{I}_{\mathrm{sat}}{ }^{-} \mathrm{I}_{0}\right)$ evaluated at $-120 \mathrm{mV}$. Data from four different oocytes were averaged.

Analysis of the proton dependency. The sequence of external solutions perfusing the ZmSUT1-expressing oocyte was the following: 1) from $\mathrm{pH} 3$ to 7 in steps of one $\mathrm{pH}$ unit in absence of external sucrose, 2) going to $\mathrm{pH} 4$, in the absence of external sucrose and finally 3) applying saturating concentration of external sucrose at $\mathrm{pH} 4$. Presteady-state currents were obtained using the standard procedure after subtraction of traces recorded at $\mathrm{pH} 4$ in saturating external sucrose. The charge $(\mathrm{Q})$ was obtained by the integral of the presteady-state currents and fitted by the Boltzmann function shown above. The slope $\mathrm{s}$ was fixed to $98 \mathrm{mV}$, the average value at $\mathrm{pH} 4$ from 20 different oocytes, see text. The maximum charge, $Q_{\max }$, was fixed at the values obtained at $\mathrm{pH} 4$; at $\mathrm{pH} 3 \mathrm{Q}_{\max }$ was considered as a free parameter of the fit and was normalized to the value at $\mathrm{pH}$. We used the relationship $\mathrm{I}=$ $\mathrm{Q} / \tau$, where $\mathrm{Q}$ and $\tau$ are the charge and the slow time constant at $\mathrm{pH} 4$ and 5 , to calculate $\mathrm{I}(\mathrm{pH}=5)$ i.e. the transported current at $\mathrm{pH}$ 5. $\mathrm{I}(\mathrm{pH}=5)$ was divided by $\mathrm{I}(\mathrm{pH}=4, \mathrm{~V}=-120 \mathrm{mV})$ and compared, as shown in Fig. 6E, with the stationary currents at $\mathrm{pH} 5$ normalized to the value of the current at $\mathrm{pH} 4$ and $\mathrm{V}=-120$. Stationary currents were obtained by a different set of experiments.

Data are given as mean \pm SEM together with the number, $n$, of different oocyte investigated.

The TECV set-up was equipped by a home-made system to control the temperature. Experiments were performed at a temperature of $22.0 \pm 0.1^{\circ} \mathrm{C}$.

\section{Supporting Information}

Figure S1 The empty symbols are the capacitance measured using the method of Adrian and Almers (1976) in response to different external sucrose $(\mathrm{A})$ or $\mathrm{pH}(\mathrm{B})$ conditions. Continuous lines were derived from the $\mathrm{Q}-\mathrm{V}$ plot of Fig. $5 \mathrm{~B}$ in $(\mathrm{A})$ and of Fig. 6B in (B).

Found at: doi:10.1371/journal.pone.0012605.s001 (4.27 MB TIF)

\section{Acknowledgments}

We are indebted with Prof. Antonio Peres (University of Insubria, Italy) for his availability, fruitful discussions and suggestions. We thank Dr. Franco Conti and Dr. Michael Pusch (IBF-GNR Italy) for useful discussions. We gratefully acknowledge Gregory Harms for critical reading and comments on the manuscript.

\section{Author Contributions}

Conceived and designed the experiments: AC HK EB RH DG. Performed the experiments: AC DG. Analyzed the data: AC DG. Wrote the paper: AC HK EB RH DG.

7. Carpaneto A, Geiger D, Bamberg E, Sauer N, Fromm J, et al. (2005) Phloemlocalized, proton-coupled sucrose carrier ZmSUT1 mediates sucrose efflux under the control of the sucrose gradient and the proton motive force. J Biol Chem 280: 21437-21443.

8. Boorer KJ, Loo DD, Frommer WB, Wright EM (1996) Transport mechanism of the cloned potato $\mathrm{H}^{+}$/sucrose cotransporter StSUT1. J Biol Chem 271: $25139-25144$.

9. Zhou J, Theodoulou F, Sauer N, Sanders D, Miller AJ (1997) A kinetic model with ordered cytoplasmic dissociation for SUC1, an Arabidopsis $\mathrm{H}^{+}$/ sucrose cotransporter expressed in Xenopus oocytes. J Membr Biol 159: 113-125.

10. Hilgemann DW, Lu CG (1999) GAT1 (GABA: $\left.\mathrm{Na}^{+}: \mathrm{Cl}^{-}\right)$cotransport function. Database reconstruction with an alternating access model. J Gen Physiol 114 : $459-475$. 
11. Loo DD, Hirayama BA, Gallardo EM, Lam JT, Turk E, et al. (1998) Conformational changes couple $\mathrm{Na}^{+}$and glucose transport. Proc Natl Acad Sci U S A 95: 7789-7794.

12. Parent L, Supplisson S, Loo DD, Wright EM (1992) Electrogenic properties of the cloned $\mathrm{Na}^{+} /$glucose cotransporter: II. A transport model under nonrapid equilibrium conditions. J Membr Biol 125: 63-79.

13. Su A, Mager S, Mayo SL, Lester HA (1996) A multi-substrate single-file model for ion-coupled transporters. Biophys J 70: 762-777.

14. Bossi E, Centinaio E, Castagna M, Giovannardi S, Vincenti S, et al. (1999) Ion binding and permeation through the lepidopteran amino acid transporter KAAT1 expressed in Xenopus oocytes. J Physiol 515(Pt 3): 729-742.

15. Mager S, Naeve J, Quick M, Labarca C, Davidson N, et al. (1993) Steady states, charge movements, and rates for a cloned GABA transporter expressed in Xenopus oocytes. Neuron 10: 177-188.

16. Loo DD, Hirayama BA, Cha A, Bezanilla F, Wright EM (2005) Perturbation analysis of the voltage-sensitive conformational changes of the $\mathrm{Na}^{+}$/glucose cotransporter. J Gen Physiol 125: 13-36.

17. Meinild A-K, Hirayama BA, Wright EM, Loo DDF (2001) Fluorescence Studies of Ligand-Induced Conformational Changes of the $\mathrm{Na}^{+} /$Glucose Cotransporter. Biochemistry 41: 1250-1258.

18. Loo DD, Hirayama BA, Karakossian MH, Meinild AK, Wright EM (2006) Conformational dynamics of hSGLT1 during $\mathrm{Na}^{+}$/glucose cotransport. J Gen Physiol 128: 701-720.

19. Armstrong CM (1975) Evidence for ionic pores in excitable membranes. Biophys J 15: 932-933.

20. Birnir B, Loo DD, Wright EM (1991) Voltage-clamp studies of the $\mathrm{Na}^{+} /$glucose cotransporter cloned from rabbit small intestine. Pflugers Arch 418: 79-85.

21. Forster I, Hernando N, Biber J, Murer H (1998) The voltage dependence of a cloned mammalian renal type II $\mathrm{Na}^{+} / \mathrm{Pi}$ cotransporter (NaPi-2). J Gen Physiol 112: 1-18.

22. Keynes RD (1994) The kinetics of voltage-gated ion channels. Q Rev Biophys 27: 339-434.

23. Klamo EM, Drew ME, Landfear SM, Kavanaugh MP (1996) Kinetics and stoichiometry of a proton/myo-inositol cotransporter. J Biol Chem 271: 14937-14943

24. Loo DD, Hazama A, Supplisson S, Turk E, Wright EM (1993) Relaxation kinetics of the $\mathrm{Na}^{+}$/glucose cotransporter. Proc Natl Acad Sci U S A 90: $5767-5771$.
25. Parent L, Supplisson S, Loo DD, Wright EM (1992) Electrogenic properties of the cloned $\mathrm{Na}^{+}$/glucose cotransporter: I. Voltage-clamp studies. J Membr Biol 125: 49-62.

26. Schmitt BM, Koepsell H (2002) An improved method for real-time monitoring of membrane capacitance in Xenopus laevis oocytes. Biophys J 82: 1345-1357.

27. Stuhmer W, Conti F, Suzuki H, Wang XD, Noda M, et al. (1989) Structural parts involved in activation and inactivation of the sodium channel. Nature 339: 597-603.

28. Adrian RH, Almers W (1976) The voltage dependence of membrane capacity. J Physiol 254: 317-338.

29. Bezanilla F (2000) The voltage sensor in voltage-dependent ion channels. Physiol Rev 80: 555-592.

30. Fesce R, Giovannardi S, Binda F, Bossi E, Peres A (2002) The relation between charge movement and transport-associated currents in the rat GABA cotransporter rGAT1. J Physiol 545: 739-750.

31. Peres A, Giovannardi S, Bossi E, Fesce R (2004) Electrophysiological insights into the mechanism of ion-coupled cotransporters. News Physiol Sci 19: 80-84.

32. Kaback HR, Dunten R, Frillingos S, Venkatesan P, Kwaw I, et al. (2007) Sitedirected alkylation and the alternating access model for LacY. Proc Natl Acad Sci U S A 104: 491-494.

33. Majumdar DS, Smirnova I, Kasho V, Nir E, Kong X, et al. (2007) Singlemolecule FRET reveals sugar-induced conformational dynamics in LacY. Proc Natl Acad Sci U S A 104: 12640-12645.

34. Nussberger S, Steel A, Trotti D, Romero MF, Boron WF, et al. (1997 Symmetry of $\mathrm{H}^{+}$binding to the intra- and extracellular side of the $\mathrm{H}^{+}$-coupled oligopeptide cotransporter PepT1. J Biol Chem 272: 7777-7785.

35. Boorer KJ, Loo DD, Wright EM (1994) Steady-state and presteady-state kinetics of the $\mathrm{H}^{+}$/hexose cotransporter (STP1) from Arabidopsis thaliana expressed in Xenopus oocytes. J Biol Chem 269: 20417-20424.

36. Cherubino F, Bossi E, Miszner A, Ghezzi C, Peres A (2010) Transient currents in the glycine cotransporter GlyT1 reveal different steps in transport mechanism. J Mol Neurosci 41: 243-251.

37. Becker D, Dreyer I, Hoth S, Reid JD, Busch H, et al. (1996) Changes in voltage activation, $\mathrm{Cs}^{+}$sensitivity, and ion permeability in $\mathrm{H} 5$ mutants of the plant $\mathrm{K}^{+}$ channel KAT1. Proc Natl Acad Sci U S A 93: 8123-8128. 